\title{
Inquérito sobre a funcionalidade do joelho em mulheres praticantes de BODYPUMPTM
} Survey knee functionality practitioners women BODYPUMP TM

\author{
Daniel Vicentini de Oliveira* \\ Cláudia Regina Leal de Souza** \\ José Roberto Andrade do Nascimento Júnior ***
}

\begin{abstract}
Resumo
Este estudo teve como objetivo analisar a funcionalidade do joelho em mulheres praticantes exclusivamente de BODYPUMPTM. Foram avaliadas 40 mulheres. O inquérito sobre a funcionalidade do joelho se deu por meio do questionário Cincinatti adaptado, que varia com escores de 0 a 100, sendo quanto mais próximo de 100, mais funcional o joelho. De uma forma geral, as mulheres apresentaram escore mediano de 88,5 de funcionalidade do joelho. Verificou-se também que a maioria das mulheres não possuía falseio no joelho (60\%), não apresentaram limitação para a realização da caminhada (65\%) e escadas (60\%). Observou-se que 45\% das mulheres não relataram ter limitação para a corrida e, em relação aos saltos e rotações, a maioria das mulheres não possuía limitação (37,5\%) ou possuía incapacidade discreta (30\%). A maioria das mulheres relatou que não sentem dor durante os exercícios do BODYPUMPTM $(52,5 \%)$, entretanto, $45 \%$ delas relataram dores durante a execução do agachamento e afundo nesta modalidade. Houve diferença significativa $(p>0,05)$ na comparação da funcionalidade do joelho das mulheres praticantes de BODYPUMPTM em função do tempo de prática, indicando que as mulheres que praticam essa modalidade há mais de cinco anos apresentaram maior escore, ou seja, melhor funcionalidade do joelho quando comparadas com as mulheres praticantes entre três meses e um ano $(p=0,048)$. Conclui- se que as mulheres praticantes de BODYPUMPTM possuem boa funcionalidade do joelho, considerado o escore máximo do instrumento utilizado.
\end{abstract}

Palavras-chave: Exercício físico. Ginástica. Capacidade funcional. Exercício de resistência.

\begin{abstract}
This study aimed to examine the knee functionality exclusively BODYPUMP TM practitioners women. 40 women were evaluated. The investigation of knee functionality was through the Cincinatti adapted questionnaire, which varies from 0 to 100 scores, and the closer to 100, more functional knee. In general, women had a median score of 88.5 of knee functionality. It was also found that most women did not have falseio knee $(60 \%)$ showed no limitation to perform the walk $(65 \%)$ and stairs $(60 \%)$. It was observed that $45 \%$ of women reported no limitation for the race and, for the jumps and spins; most women did not have limitation (37.5\%) or had mild disability (30\%). Most women reported that they do not feel pain during the BODYPUMP TM exercises (52.5\%), however, $45 \%$ reported pain during the execution of the squat and sink in this mode. There was a significant difference ( $p>0.05)$ in the comparison of the knee of BODYPUMP TM practitioners women functionality due to the practice time, indicating that women who practice this sport for more than five years had higher scores, ie better knee functionality when compared to women practitioners between three months and one year $(p=0.048)$. It is concluded that BODYPUMP TM practitioner's women have good functionality of the knee, considered the highest score of the instrument used.
\end{abstract}

Keywords: Physical exercise. Fitness. Functional capacity. Resistance exercise.

DOI: 10.15343/0104-7809.20164002199207

*Universidade Estadual de Camplnas (UNICAMP), Campinas, SP. Faculdade Metropolitana de Maringá (FAMMA), Maringá - PR, Brasil.

** Departamento de Educação Física (bacharelado) da Faculdade Metropolitana de Maringá (FAMMA). Maringá - PR, Brasil.

*** Departamento de Educação Física da Universidade Federal do Vale do São Francisco (UNIVASF). Petrolina - PE, Brasil.

Os autores declaram não haver conflitos de interesse. 


\section{INTRODUÇÃO}

O joelho é uma articulação muito requisitada pelo corpo humano, pelo fato de ser utilizado como pivô, independentemente da prática desportiva, sendo, portanto, alvo de dores, inchaços, crepitações, comprometendo a funcionalidade do mesmo. Esta articulação é projetada para mobilidade, fornecida pela estrutura óssea, estabilidade, fornecida pelos tecidos moles (ligamentos, músculos e cartilagens) ${ }^{1}$. Muitas atividades diárias exigem considerável flexão e extensão de joelho, como caminhar, subir e descer escadas, além de suportar grandes cargas ${ }^{2}$.

Dores e a diminuição da funcionalidade do joelho são comuns tanto em pessoas ativas como em sedentárias, sendo as causas multifatoriais como o repetido esforço de forma irregular, desgastes, subluxações, desequilíbrios musculares, incompatibilidade entre aplicação de sobrecarga e capacidade de recuperação e pré-disposição genética. Todos esses fatores podem contribuir tanto para o aumento da dor, como pode desenvolver uma lesão definitiva. Qualquer desconforto no joelho pode desestabilizar a articulação, causando uma diminuição da função ${ }^{3}$.

O joelho é uma articulação de grande instabilidade, expondo essa articulação diante de atividades que exigem grande mobilidade a maior número de traumas, resultando em dor, fraqueza muscular e até perda de função ${ }^{4,5}$. Tais traumas podem ser provocados por um excesso das limitações fisiológicas, ou bem por um estresse de intensidade normal sobre músculo enfraquecido ou não treinado, gerando insegurança, desconforto e a dor ${ }^{2}$.

As mulheres são mais acometidas a desenvolverem um processo de algum tipo de desconforto nos joelhos, pela sua anatomia, diferenças neuromusculares e variações hormonais. Visto que as mulheres apresentam o desequilíbrio muscular como principal fator etiológico ${ }^{6}$. Para isso, programas de fortalecimento muscular são propostos por meio de treinamento resistido (TR) para a melhora da dor nos joelhos e da capacidade funcional?.

As adaptações induzidas pelo TR em mulheres estão bem estabelecidas na literatura científica e isso tem levado à sua utilização tanto em mulheres saudáveis como aquelas com debilidades, que sofrem com dores no joelho, e algumas já lesionadas ${ }^{8}$. A dor e a diminuição de função que ocorrem nos joelhos estão diretamente correlacionadas com a diminuição de força do quadríceps. A literatura defende que o TR é eficiente para o controle sintomático e para a melhora funcional, mesmo em casos mais avançados ${ }^{9}$.

O TR pode melhorar a capacidade funcional do joelho, porém, se realizados de forma inadequada, sem supervisão, fora do alinhamento, com uma sobrecarga que não respeite a individualidade biológica do indivíduo e em excessos, podem gerar o efeito contrário, em longo prazo, que é a limitação do mesmo nas atividades funcionais ${ }^{10}$. Agre, Rasmussen e Miller ${ }^{11}$ citam a importância do ganho de força nos músculos que envolvem a articulação do joelho, entretanto, muitas vezes os exercícios para tal objetivo podem ocasionar desgastes e até traumas pela própria execução dos mesmos.

Um modelo de treinamento resistido que faz sucesso entre as mulheres, principalmente àquelas que não são adeptas a musculação, é o programa BODYPUMPTM. Desenvolvido pela empresa Les Mills Internacional ${ }^{\mathrm{TM}}$, na Nova Zelândia em 1990, por uma equipe de médicos, fisioterapeutas, fisiologistas, biomecânicos e profissionais de educação física. O BODYPUMPTM chegou ao Brasil em 1997, por meio de franquia, sendo representada pela empresa Body Systems ${ }^{\circledR}$, que no ano de 2015 começou a fazer parte da Les Mills Brasil囚. Sucesso por ser uma aula coletiva, ao som de músicas que empolgam e incentivam ao treino coreografado, o BODYPUMPTM utiliza- se de cargas extras, com barras e anilhas, objetivando o fortalecimento e definição muscular, com exercícios de treinamento de força e resistência, dentro da sala de ginástica.

\section{MÉTODO}

A presente pesquisa, realizada entre junho a dezembro de 2015, de abordagem quantitativa, descritiva, observacional e transversal, foi aprovada pelo Comitê de Ética em Pesquisa do Centro Universitário Cesumar (UNICESUMAR) 
por meio do parecer número 1.157.134/2015. Foram seguidos os preceitos éticos da Resolução 196/96 do Conselho Nacional de Saúde do Ministério da Saúde do Brasil, que regulamenta as pesquisas envolvendo seres humanos.

Para realização desse estudo, foi feito um levantamento no site da Les Mills Brasil $®$, das academias franquiadas a mesma, no município de Maringá, estado do Paraná. Foram constatadas nove academias franquiadas, que possuem o programa BODYPUMPTM. A população foi composta de mulheres praticantes de BODYPUMPTM destas nove academias.

Foram incluídas as mulheres praticantes exclusivamente da modalidade, a no mínimo três meses, com frequência mínima de duas vezes semanais, com idade igual ou superior a 20 anos, com no máximo 50 anos. Foram excluídos os praticantes do sexo masculino, pois constatou-se baixa prevalência dos mesmos nas aulas. A amostra final foi composta de 40 mulheres.

Para a caracterização das mulheres foi utilizado um questionário composto por informações referentes à idade, estado civil, situação ocupacional, frequência semanal, tempo de prática de ginástica, se praticam outra modalidade além do BODYPUMPTM e há quanto tempo praticam as mesmas.

A avaliação funcional do joelho se deu por meio do questionário adaptado, desenvolvido em Cincinatti, adaptado por Noyes ${ }^{12}$, que atribui notas a diversos itens relacionados à dor, falseio capacidade de deslocamento, nível global de atividade física, com escores de 0 a 100 , sendo o joelho normal contemplado com 100 pontos.

A coleta das informações somente ocorreu após a colaboradora aceitar participar da pesquisa através da leitura, explicação e assinatura do Termo de Consentimento Livre e Esclarecido (TCLE).

Foram entregues os questionários em data previamente agendada pela academia. As participantes levaram os questionários para casa, com data já estabelecida para devolução e recolhida pelo próprio professor do horário.

Para a análise dos dados, foi utilizado frequência e percentual para as variáveis categóricas. Para as variáveis numéricas, inicialmente foi verificada a normalidade dos dados por meio do teste de Shapiro-Wilk. Como os dados não apresentaram distribuição normal, foram utilizadas Mediana (Md) e Quartis (Q1; Q3) para a caracterização dos resultados. Na comparação do escore da avaliação funcional do joelho em função da frequência semanal, foi utilizado o teste " $U$ " de Mann-Whitney; e do tempo de prática, foi utilizado o teste de Kruskal-Wallis, após a verificação da não homogeneidade dos dados, seguido do teste " $U$ " de Mann-Whitney para pares de grupos. Para analisar a associação da funcionalidade do joelho com a faixa etária e tempo de prática efetuou-se o Teste de Qui-quadrado. A significância adotada foi de $p<0,05$.

\section{RESULTADOS}

Os dados referentes à distribuição de frequência do perfil das mulheres praticantes de BODYPUMPTM estão expostos na tabela 1.

Tabela 1- Distribuição de frequência do perfil das mulheres praticantes de BODYPUMPTM da cidade de Maringá-PR, Brasil, de junho a dezembro/2015.

\begin{tabular}{lcc}
\hline \multicolumn{1}{c}{ VARIÁVEIS } & $f$ & $\%$ \\
\hline Faixa Etária & & \\
\hline 20 a 30 anos & 7 & 67,5 \\
31 a 40 anos & 6 & 17,5 \\
41 a 50 anos & & \\
\hline Frequência Semanal & 34 & 85,0 \\
\hline 1 a 2 vezes por semana & 06 & 15,0 \\
3 vezes por semana & & \\
\hline Tempo de Prática & 24 & 60,0 \\
\hline 3 meses a 1 ano & 10 & 25,0 \\
1 a 5 anos & 06 & 15,0 \\
Mais de 5 anos & & \\
\hline Modalidade praticada anteriormente & 22 & 55,0 \\
\hline Esporte & 08 & 20,0
\end{tabular}


...continuação - Tabela 1

202 ...continuação - Tabela 2

\begin{tabular}{lcc}
\hline Escadas & 24 & 60,0 \\
\hline Sem limitação & 10 & 25,0 \\
Incapacidade discreta & 06 & 15,0 \\
\hline Incapacidade moderada & & \\
\hline Corrida & 18 & 45,0 \\
\hline Sem limitação & 09 & 22,5 \\
Incapacidade discreta & 13 & 32,5 \\
\hline Incapacidade moderada & & \\
\hline Saltos e Rotações & 15 & 37,5 \\
\hline Sem limitação & 12 & 30,0 \\
Incapacidade discreta & 06 & 15,0 \\
Incapacidade moderada & 07 & 17,5 \\
\hline Incapacidade intensa & & \\
\hline Sente dor durante a aula & 21 & 52,5 \\
\hline Não sente dor & 09 & 22,5 \\
Durante agachamento & 09 & 22,5 \\
Durante afundo & 01 & 2,5 \\
\hline Em qualquer exercício & &
\end{tabular}

A maioria das mulheres não apresentou limitação para a realização da caminhada $(65,0 \%)$ e subir e descer escadas $(60,0 \%)$. Em relação a corrida, observou-se que $45,0 \%$ das mulheres não relataram ter limitação e que $32,5 \%$ relataram incapacidade moderada.

Verificou-se também (Tabela 2) que, em relação aos saltos e rotações, a maioria das mulheres não possui limitação $(37,5 \%)$ ou possui incapacidade discreta $(30,0 \%)$. A maioria das mulheres relatou que não sentem dor durante os exercícios do BODYPUMPTM (52,5\%), entretanto, $(45,0 \%)$ das mulheres relataram dores durante a execução do agachamento e afundo nesta modalidade.

A tabela 3 apresenta a comparação da funcionalidade do joelho das mulheres praticantes de BODYPUMPTM por meio do escore de Cincinatti em função da frequência semanal e tempo de prática. 
Tabela 3- Comparação da funcionalidade do joelho das mulheres praticantes de BODYPUMPTM por meio do escore de Cincinatti em função da frequência semanal e tempo de prática. Cidade de Maringá-PR, Brasil, de junho a dezembro/2015.

\begin{tabular}{llcc}
\hline \multicolumn{1}{c}{ VARIÁVEIS } & Md & Q1-Q3 & P \\
\hline $\begin{array}{l}\text { Tempo } \\
\text { de Prática }\end{array}$ & & & \\
\hline $3{\text { meses a 1 } \text { ano }^{\text {a }}}$ & 74,0 & $47,00-100,00$ & \\
1 a 5 anos & 95,5 & $83,75-100,00$ & $0,047^{*}$ \\
Mais de 5 anos $^{\text {a }}$ & 99,0 & $93,75-100,00$ & \\
\hline $\begin{array}{l}\text { Frequência } \\
\text { Semanal }\end{array}$ & & & \\
\hline $\begin{array}{l}\text { 1 a } 2 \text { vezes } \\
\text { por semana }\end{array}$ & 92,5 & $73,50-100,00$ & \\
$\begin{array}{l}\text { 3 vezes } \\
\text { por semana }\end{array}$ & 47,0 & $46,50-100,00$ & 0,197 \\
\hline
\end{tabular}

*Diferença significativa: $p<0,05$ - ${ }^{\text {TTeste de }}$ Kruskal-Wallis (tempo de prática) e b" $U$ " de Mann-Whitnney (frequência semanal) entre: a) 3 meses a ano e Mais de 5 anos $(p=0,048)$.

Houve diferença significativa $(p>0,05)$ na comparação da funcionalidade do joelho das mulheres praticantes de BODYPUMP ${ }^{\mathrm{TM}}$ por meio do escore de Cincinatti em função tempo de prática (Tabela 3 ), indicando que as mulheres que praticam o BODYPUMPTM há mais de 5 anos apresentaram maior escore, ou seja, funcionalidade do joelho, quando comparadas com as mulheres praticantes entre 3 meses e 1 ano $(p=0,048)$.

\section{DISCUSSÃO}

Nota-se (Tabela 1) a prevalência de mulheres entre 20 e 30 anos (67,5\%). Segundo o manual do professor, as aulas de BODYPUMPTM podem ser frequentadas por indivíduos com 15 anos ou mais, respeitando as limitações físicas, adequando as cargas para o aluno iniciante até o mais preparado ${ }^{13}$. A maioria das mulheres pratica o programa BODYPUMPTM há menos de um ano $(60 \%)$ e com frequência de prática de duas vezes por semana (85\%). O ideal é que se pratique a modalidade três vezes por semana, desde que tenha um descanso muscular de 24 horas. Entretanto, por uma questão de melhor distribuição de aulas, as academias normalmente oferecem duas aulas semanais.

Observou-se que a maioria das mulheres considera estar com peso normal $(80 \%)$ mas tem-se que levar em consideração também aquelas que possuem autopercepção do corpo como acima do peso, e se isto é real, pois as questões relacionadas à imagem corporal estão se tornando mais e mais evidente no campo da saúde e da influência forma o sujeito constrói a sua identidade, bem como a maneira como eles percebem o seu próprio corpo e que eles vêem tão saudável. A constante transformação do corpo, para inserção em um padrão social imposto pela mídia, revela uma insatisfação pessoal, adestrado pela cultura, concebido socialmente, alterado segundo crenças e ideais coletivamente estabelecidos, vivendo assim uma obsessão de ter um corpo estereotipado ${ }^{14}$.

Mais da metade das mulheres avaliadas $(75 \%)$ relataram ter, com a prática da modalidade, o objetivo de definição muscular. O programa BODYPUMPTM é uma modalidade que preconiza a definição muscular, por meio de exercícios para vários grupos musculares, com altas repetições e cargas moderadas. Muitas mulheres não procuram a musculação, ou não treinam com altas cargas, pois tem receio de ficarem "masculinizadas" buscando, portanto, no BODYPUMPTM a modalidade ideal, que fortalece e define. Santos, Nascimento e Liberali ${ }^{15}$ citam em seu trabalho que o treinamento de resistência muscular é uma ótima opção para quem já está no peso ideal e busca tonificar os músculos, sem hipertrofia significativa, mas com resultados satisfatórios na definição muscular.

O conceito de equilíbrio funcional musculotendíneo reflete um parâmetro importante na funcionalidade do joelho. A avaliação isocinética tem sido usada como método para se determinar o padrão funcional da força e do equilíbrio muscular. A relação entre músculo agonista e antagonista é uma forma adequada para saber se existe proporção e consequentemente o equilíbrio muscular ${ }^{16}$.

O estudo de Shinzato et al. ${ }^{17}$ mostrou que indivíduos com insuficiência de ligamento 
cruzado anterior (LCA) com tempo médio de 2,7 anos, a relação da musculatura flexora e extensora do joelho era de $66 \%$ e $82 \%$ nas velocidades, respectivamente, de $60^{\circ} / \mathrm{seg}$ e $180^{\circ} / \mathrm{seg}$. Os autores descrevem que ocorre desequilíbrio muscular, com déficit de força da musculatura do joelho com insuficiência de das causas do sintoma de falseio do joelho, porém a insuficiência ligamentar não foi uma variável pesquisada no presente estudo. Supõese, então, que a prática de BODYPUMPTM por meio de exercícios de força e resistência muscular, seja uma aliada a estabilidade do joelho, e, portanto, possa estar provocando a positividade dos dados apresentados.

Em relação à limitação no nível global das atividades, 18 mulheres (45\%), não apresentaram limitação, entretanto 15 delas $(37,5 \%)$ relataram queda no desempenho em atividades vigorosas. A capacidade de realizar as tarefas do dia a dia, o qual envolve diretamente a capacidade funcional do joelho pode ser bastante prejudicada conforme visto nesse resultado. Segundo Garver et al. ${ }^{18}$, manter a mobilidade articular é muito importante para as atividades da vida diária, pois a dor leva a perda de amplitude de movimento, causando encurtamento, contratura em músculos e estruturas capsulares, podendo dificultar a funcionalidade do joelho.

No estudo de Cabral ${ }^{19}$ com 40 mulheres sedentárias com idade entre 18 e 32 anos, foi aplicado questionário de Lysholm (escala de capacidade funcional do joelho). Foram realizados exercícios para fortalecimento do quadríceps em cadeia cinética fechada (exercícios realizados com os membros inferiores fixos) como o agachamento, com aumento progressivo de carga. O trabalho durou oito semanas, com frequência de duas vezes semanais. Os resultados mostraram uma melhora na intensidade da dor assim como também na capacidade funcional relacionada ao joelho.

A maioria das mulheres não apresentou limitação para a realização da caminhada $(65,0 \%)$ e subir e descer escadas $(60,0 \%)$. O caminhar, subir e descer escadas são atividades da vida diária do homem sendo o caminhar um ciclo dividido em apoio e balanço. O subir

e descer escadas são atividades complexas que demandam do adequado sinergismo e estabilidade muscular ${ }^{20}$.

O LCA é o principal ligamento que segura a translação anterior da tíbia sobre o fêmur, sendo que se algumas alterações dessas estruturas estiverem danificadas ou associadas a diminuição da propriocepção, alteração dos reflexos musculares ou diminuição de força muscular do quadríceps, comprometerá a funcionalidade do joelho, ou seja, limitando o indivíduo a atividades da vida diária. Considerando-se as sobrecargas a que o sistema musculoesquelético é exposto, estas podem vir a causar prejuízos à estrutura e ao funcionamento do aparelho locomotor ${ }^{21}$.

Segundo Baraúna e Cheik $^{22}$, durante a marcha, ou subir e descer escadas, o estresse na cartilagem articular do joelho é muito maior do que o considerado unicamente pelo peso do corpo, sobrecarregando o compartimento medial, comprometendo a articulação.

A ação de o joelho no caminhar, subir e descer escadas está diretamente ligada aos movimentos executados nas aulas de BODYPUMPTM Como o agachamento e afundo. O programa BODYPUMPTM trabalha com amplitudes seguras, ou seja, até 90 graus de flexão, que protege a articulação do joelho de dores que eventualmente ocorreriam por um agachamento total.

Em relação à corrida, observou-se que $45 \%$ das mulheres não relataram ter limitação e que $32,5 \%$ relataram incapacidade moderada. $A$ corrida envolve integração de movimentos em diferentes articulações e segmentos do corpo, que inclui o ângulo do tronco, balanços dos braços, comprimento do passo, levantamento da perna de trás e por fim o padrão de aterrissagem do pé no solo ${ }^{23}$. Segundo Dierks et al. ${ }^{24}$, o que mais a literatura traz no sentido de ser o fator mais agravante é o momento da aterrissagem do pé, pois se acredita que as forças verticais de reação ao solo que são transmitidas para os membros inferiores a cada toque do pé durante corrida seja a principal causa de comprometimento da articulação do joelho.

Em relação aos saltos e rotações, a maioria das mulheres não possui limitação $(37,5 \%)$ ou possui incapacidade discreta $(30,0 \%)$. O salto 
começa com a impulsão passando para o voo, finalizando na distância de aterrissagem. O mau alinhamento dinâmico dos membros inferiores pode ser a causa para desequilíbrios e dores musculares do joelho no momento da aterrissagem. Quanto maior a força vertical exercida sobre a superfície de impulsão, maior será a força vertical de reação disponível para elevar o indivíduo, ou seja, maior pressão sobre os joelhos ${ }^{25}$. A potência muscular dos membros inferiores é importante, portanto, para o desempenho do salto. A potência é definida como a realização do trabalho por unidade de tempo ${ }^{26}$.

O funcionamento muscular é caracterizado pela produção de força e de potência, mas também pela capacidade de manter a força e a potência ao longo de um determinado período de tempo o que acarreta em resistência. Para Fleck e Kraemer $^{6}$, o desenvolvimento da potência está relacionado ao desempenho da maioria das atividades da vida diária. O programa BODYPUMPTM trabalha potência por meio de movimentos de cadencias diferenciadas. No agachamento livre, por exemplo, a música tem em média seis minutos, com aproximadamente 124 execuções em diferentes cadencias, vencendo a estabilidade, o equilíbrio, ganhando resistência e potência.

A maioria das mulheres relatou que não sentem dor durante os exercícios do BODYPUMP'M $(52,5 \%)$, entretanto, $45 \%$ das mulheres relataram dores durante a execução do agachamento e afundo nesta modalidade. A dor é um sintoma subjetivo que varia nas características de acordo com quem a descreve. Uma das maneiras que se tem para identificar a presença de dor é a aplicação de instrumentos avaliativos o qual a própria pessoa é quem faz seu relato, sendo o questionário Cincinatti um desses instrumentos.

A presença da dor é um sinal de defesa do corpo humano já que as articulações têm células que avisam o cérebro quando elas estão sendo exigidas demais. Agachamentos e afundos são exercícios importantes e têm sido alvo de inúmeros estudos. Entretanto, como em qualquer outro exercício, se realizado de forma incorreta ou excessiva, o agachamento pode resultar em dor.

As dores internas da articulação do joelho são de inúmeras causas, com consequências variadas para a função e a qualidade funcional dos indivíduos. Sobrepeso, idade, enfraquecimento dos músculos quadríceps, encurtamento dos isquiotibiais, questões anatômicas e a própria genética são conducentes a lesões articulares, as quais dão seus sinais através da dor. Porém, para quase todos os casos, a literatura traz como tratamento para as disfunções do joelho, o fortalecimento e alongamento dos músculos do quadríceps e dos isquiotibiais ${ }^{27}$.

O treinamento muscular deve ter como objetivos promover o aumento de força de contração do músculo vasto medial oblíquo de maneira concêntrica e excêntrica durante toda a amplitude de movimento. Os exercícios isométricos são conhecidos como um meio eficaz para se alcançar o fortalecimento muscular, sendo usados em todo o processo da reabilitação. Esses exercícios de certa forma aumentam a força muscular, porém, esses ganhos são específicos, parecendo ocorrer uma melhora em $20 \%$ na posição articular em que é realizado o exercício ${ }^{28}$.

Houve diferença significativa $(p>0,05)$ na comparação da funcionalidade do joelho das mulheres praticantes de BODYPUMPTM por meio do escore de Cincinatti em função tempo de prática (Tabela 3), indicando que as mulheres que praticam o BODYPUMPTM há mais de 5 anos apresentaram maior escore, ou seja, funcionalidade do joelho, quando comparadas com as mulheres praticantes entre 3 meses e 1 ano $(p=0,048)$, o que supostamente nos remete a ideia de que os exercícios de fortalecimento com sobrecarga, como os realizados no BODYPUMPTM, podem melhorar a força musculotendíneo, melhorando a funcionalidade do joelho, obtendo benefícios dessa funcionalidade para outras atividades físicas, bem como as atividades da vida diária como pegar algo no chão, sentar, levantar, subir e descer de uma cadeira ou escadas ${ }^{29}$. Entretanto, o número de mulheres que sentem dor, ou falseio no joelho pode ser explicado pelo desequilíbrio muscular, uma vez que BODYPUMPTM tem maior foco no fortalecimento do quadríceps por meio do agachamento, do que da musculatura dos ísquiotibiais.

O estudo apresenta algumas limitações 
como a ausência de um grupo controle constituídas por mulheres não praticantes de exercícios, assim como de um grupo de praticantes de outros programas de ginástica. Outro aspecto que deve ser destacado referese ao histórico de atividades anteriores, ou extras do seu cotidiano, fato que pode resultar em diferentes mensurações. Entretanto, mesmo com as limitações apontadas, esta pesquisa poderá fornecer aos profissionais da saúde importantes conhecimentos sobre a importância de incentivar o fortalecimento dos joelhos, independente de qual a prática desportiva, até mesmo de quem não pratica nenhuma atividade, a fim de manter o equilíbrio e saúde dos mesmos, fato necessário para boa funcionalidade, promoção da independência e qualidade de vida.

\section{CONCLUSÃO}

Conclui-se que a maioria das mulheres praticantes do programa BODYPUMPTM, analisadas nessa pesquisa tem uma boa funcionalidade do joelho, considerado o escore máximo do instrumento utilizado. Além disso, buscam com a prática da modalidade, o objetivo de definição muscular e que a boa funcionalidade do joelho está ligada ao tempo de prática.

Verificou-se que além da boa funcionalidade do joelho, existe também em menor proporção, presença de dor durante o agachamento e afundo, o que pode ser causado pelo desequilíbrio muscular, já que a modalidade fortalece mais os músculos do quadríceps em relação aos isquiotibiais. Diante disso, enfatiza-se a importância da detecção precoce do menor sinal doloroso por parte dos profissionais de educação física, por meio de avaliações específicas para a prevenção, além de encaminhamentos a outros profissionais da saúde quando necessário.

\section{REFERÊNCIAS}

\footnotetext{
1. Messier SP, Mihalko SL, Legault C, Miller GD, Nicklas BJ, DeVita P, et al. Effects of intensive diet and exercise on kenn joint loads, inflammation and clinical outcomes among overweight and obese adults with knee osteoarthritis. Jama, 2013;310(12):923-933.

2. Cheng CK, McClean CJ, Lai YS, Chen WC, Huang CH, Lin KJ et al. Biomechanical considerations in the design of high-flexion total knee replacements. The Scientific World Journal, 2014;(2014):1-6.

3. Kim K, Jeon K. Comparisons of knee and ankle joint angles and ground reaction force according to functional differences during single-leg drop landing. J Phys Ther Sci. 2016;28:1150-1154.

4. Sinsurin K, Vachalathiti R, Jalayondeja W, Limroongreungrat W. Different sagittal angles and moments of lower extremity joints during single-leg jump landing among various directions in basketball and volleyball athletes. J Phys Ther Sci, 2013:25(9): 1109-1113. 5 Batista G. Análise da estimativa de risco da lesão do joelho de saltador em atletas de base de voleibol, Trabalho de Conclusão de Curso, UTP, Curitiba, 2002.

6. Fleck S, Kraemer W. Fundamentos do treinamento de força muscular. Porto Alegre: Ed. Artmed, 2008.

7. Lustosa LP, Silva JP, Coelho FM, Pereira DS, Parentoni AN, Pereira LSM. Efeito de um programa de resistência muscular na capacidade funcional e na força muscular dos extensores do joelho em idosas pré-frágeis da comunidade: ensaio clínico aleatorizado do tipo crossover. Rev Bras Fisioter, 2011;15(4): 318-324.

8. Mezzaroba PV, Ribeiro MS, Machado FA. Comparação de dois métodos de treinamento contra resistência na força, antropometria e composição corporal de mulheres jovens. R Bras Ci e Mov, 2014;22(2): 107-113.

9. Dadalto TV, Souza CP, Silva EB. Eletroestimulação neuromuscular, exercícios contrarresistência, força muscular, dor e função motora em pacientes com osteoartrite primária de joelho. Fisioter Mov, 2013;26(4):777-789.

11. Agree EM, Rasmussen H, Miller J. Relationships between leg power, leg strenght, knee valgus and trunk endurance with hip pain in dancers. Intern J Exerc Scien, 2015;2(7).

12. Noyes FR, McGinniss GH, Groos ES. The variable functional disability of the anterior cruciate ligament deficient knee. Orthop Clin North Am, 1985;16(1):47-67.

13. Les Mills Body Training Systems. Manual Professor Body Pump: Les Mills, 2015.

14. Silva MLA, Taquette ST, Coutinho ESF. Senses of body image in adolescentes in elementar school. Rev Saúde Pública. 2014;48(3):438444.

15. Santos VHA, Nascimento WF, Liberali R. O treinamento de resistência muscular localizada com intervenção no emagrecimento. Rev Bras Obes Nutri Emagrec, 2008;2(7):34-43.

16. Jacopetti M, Pasquini A, Constantino C. Evaluation of strength muscle recovery with isokinetic, squat jump and stiffness tests in
} 
athletes with ACL reconstruction: a case control study. Acta Biomed. 2016;6(87):76-80.

17. Shinzato GT, Vasconcelos JCP, Ogawa CT, Sampaio ICSP, Gonçalves A, Neves EM. Protocolo de avaliação funcional de joelho em patologias ortopédicas. Acta Fisiátrica, 1996;3(1):30-36.

18. Garver MJ, Focht BC, Dials J, Rose M, Lucas AR, Devor ST, Emert CF, Hackshaw KV, Rejeski WJ. Weight status and diferences im mobility performance, pain symptoms and physical activity in older, knee osteoarthritis patients. Hindawi Publish Corporat, 2014:1-7. 19. Cabral R. Lesões no Joelho em Atletas de Handebol: estudo em atletas universitários participantes dos jogos universitários brasileiros. Rev Trein Desp, 2008;9(1):8- 24.

20. Bonfim TR, Barela JA. Controle postural após a reconstrução do ligamento anterior. Rev Fis Pesq, 2004;11(1):11-18.

21. Sacco, ICN, Tessutti VD, Aliberti S, Hamamoto NA, Gomes DR, Costa MSX. Força reação do solo durante a marcha com uso do tênis e sandália plataforma. Rev Fisiot Mov, 2007;20(3):55-62.

22. Baraúna MA, Cheik NC. Obesidade e sua correlação com a osteoartrite de joelho em mulheres. Rev Fisiot Mov, 2008;21(2):93-97.

23. Silva SRD, Fraga CHW, Gonçalves M. Efeito da fadiga muscular na biomecânica da corrida: uma revisão. Motriz, 2007;13(3):225235.

24. Dierks TA, Manal KT, Hamill J, Davis IS. Proximal and distal influences on hip and knee kinematics in runners with patellofemoral pain during a prolonged run. J Orthop Sports Physic Ter, 2008;38(8):448-456.

25. Mackenzie SJ, Lavers RJ, Wallace BB. A biomechanical comparision of the vertical jump, power clean and jump squat. J Sports Scien, 2014;32(16):1576-1585.

26. Sawyer, BJ, Stokes DG, Womack CJ, Morton RH, Weltman A, Gaesser GA. Strength Training Increases Endurance Time to Exhaustion During High-Intensity Exercise Despite No Change in Critical Power. J Strength Condition Res, 2014;28(3):601-609.

27. Favarini RA, Lustosa LP. Análise da eficácia do taping patelar associado a um programa de treinamento fisioterapêutico em indivíduos do sexo feminino com disfunção patelofemoral. Fisioter Bras, 2007;8(1):09-13.

28. Allen T, Jones T, Tsay A, Morgan D, Proske U. Factors influencing muscle damage from isometric exercise. Br J Sports Med, 2014;48(7):561.

29. Garcia FHA, Mansur LL. Habilidades funcionais de comunicação: idoso saudável. Acta Fisiatrica, 2006;13(2):87-89.

Recebido em 15 de dezembro de 2015. Aprovado em 01 de junho de 2016. 